\title{
Impact of appropriate empirical antibiotics on clinical outcomes in Klebsiella pneumoniae bacteraemia
}

\author{
MY Man *, HP Shum, KC Li, WW Yan
}

\section{A B S T R A C T}

Introduction: Klebsiella pneumoniae infections can cause septic shock, multiorgan failure, and death.

Methods: This retrospective cohort study included adults with K pneumoniae bacteraemia treated from 1 January 2009 to 30 June 2017. Demographics, microbiology, and outcomes were analysed. The primary outcome was 90-day all-cause mortality; secondary outcomes were intensive care unit (ICU) and hospital mortalities, ICU and hospital lengths of stay, and ICU ventilator duration.

Results: In total, 984 patients had $K$ pneumoniae bacteraemia; of them, 686 received appropriate empirical antibiotics. Overall, 205 patients required intensive care. Older age (odds ratio $[\mathrm{OR}]=1.60$; 95\% confidence interval $[\mathrm{CI}]=1.120-2.295 ; \mathrm{P}=0.010)$, chronic kidney disease $(\mathrm{OR}=1.81 ; 95 \% \mathrm{CI}=1.181$ 2.785; $\mathrm{P}=0.007)$, mechanical ventilation $(\mathrm{OR}=1.79$; 95\% $\quad \mathrm{CI}=1.188-2.681 ; \quad \mathrm{P}=0.005), \quad$ pneumonia $(\mathrm{OR}=1.50 ; \quad 95 \% \quad \mathrm{CI}=1.030-2.187 ; \quad \mathrm{P}=0.034)$, and carbapenem-resistant or extended-spectrum betalactamase $(\mathrm{ESBL})$-producing isolates $(\mathrm{OR}=12.51$; 95\% $\mathrm{CI}=7.886-19.487 ; \mathrm{P}<0.001)$ were associated with greater risk of inappropriate empirical treatment. Ninety-day mortality was significantly higher among patients with inappropriate empirical treatment; independent predictors included pneumonia (hazard

This article was published on 13 Aug 2021 at www.hkmj.org. ratio $[\mathrm{HR}]=2.94 ; 95 \% \mathrm{CI}=2.271-3.808 ; \mathrm{P}<0.001)$, gastrointestinal infection $(\mathrm{HR}=2.77 ; 95 \% \mathrm{CI}=2.055$ -
3.744; $\mathrm{P}<0.001)$, failed empirical antibiotics $(\mathrm{HR}=2.45 ; \quad 95 \% \quad \mathrm{CI}=1.928-3.124 ; \mathrm{P}<0.001)$, older age $(\mathrm{HR}=1.79 ; 95 \% \mathrm{CI}=1.356-2.371 ; \mathrm{P}<0.001)$, solid tumour $(\mathrm{HR}=1.77 ; 95 \% \mathrm{CI}=1.401-2.231 ; \mathrm{P}<0.001)$, carbapenem-resistant or ESBL-producing isolates $(\mathrm{HR}=1.64 ; 95 \% \mathrm{CI}=1.170-2.297 ; \mathrm{P}=0.004)$, patients admitted through the Department of Medicine $(\mathrm{HR}=1.39 ; 95 \% \mathrm{CI}=1.076-1.800 ; \mathrm{P}=0.012)$, and higher total Sequential Organ Failure Assessment score $(\mathrm{HR}=1.09 ; 95 \% \mathrm{CI}=1.058-1.112 ; \mathrm{P}=0.023)$. Among ICU patients, inappropriate empirical antibiotic treatment was significantly associated with increased ventilator duration and 90-day mortality.

Conclusions: Klebsiella pneumoniae bacteraemia was associated with high 90-day and ICU mortalities; 90-day mortality increased with inappropriate empirical antibiotic treatment.

\section{Hong Kong Med J 2021;27:247-57 \\ https://doi.org/10.12809/hkmj208698}

MY Man *, MB, BS, FHKAM (Medicine)

HP Shum, MB, BS, MD

KC Li, MB, ChB

WW Yan, MB, BS, FHKAM (Medicine)

Department of Intensive Care, Pamela Youde Nethersole Eastern Hospital, Hong Kong

* Corresponding author: mayman729@gmail.com

New knowledge added by this study

- This large study of Klebsiella pneumoniae bacteraemia among patients in Hong Kong and South East Asia showed that 90-day mortality was significantly higher in patients who received inappropriate empirical treatment.

- Intensive care unit subgroup analysis showed that inappropriate empirical treatment was the strongest predictor of 90-day mortality in critically ill patients.

- Independent predictors for inappropriate empirical treatment were older age, chronic kidney disease, mechanical ventilation, pneumonia, and either carbapenem resistance or extended-spectrum beta-lactamase production.

Implications for clinical practice or policy

- Extensive efforts are needed to facilitate early, appropriate use of empirical antibiotics, including the use of a current antibiogram, implementation of multidisciplinary sepsis management guidelines, and establishment of protocols among pharmacists, microbiologists, clinicians, and nurses.

- Careful assessment of empirical antibiotic treatment may be warranted in patients with pneumonia, gastrointestinal infection, failed empirical antibiotics, older age, solid tumour, patients admitted through the Department of Medicine, and/or higher total Sequential Organ Failure Assessment score. 


\section{適當的經驗性抗生素對肺炎克雷伯菌血症臨床 結果的影響 \\ 文敏儀、沈海平、李健超、殷榮華}

引言：肺炎克雷伯菌感染可導致感染性休克、多重器官衰竭及死亡。

方法：這項回顧性隊列研究納入2009年1月1日至2017年6月30日期 間接受治療的肺炎克雷伯菌血症成人患者。分析人口統計學、微生物 學和結局。主要結果為90天全因死亡率；次要結果包括重症監護病房

( ICU ) 死亡率和醫院死亡率、ICU逗留時間和住院時間, 以及患者 使用ICU呼吸機時間。

結果：肺炎克雷伯菌血症共984例；其中686例接受適當的經驗性抗 生素治療。共205名患者需要重症監護。年齡較大（比值比 $=1.60$; 95\%置信區間 $=1.120-2.295 ； \mathrm{P}=0.010)$ 、慢性腎病（比值比 $=1.81 ；$ $95 \%$ 置信區間 $=1.181-2.785 ; \mathrm{P}=0.007$ ）、機械通氣（比值比 $=1.79$; $95 \%$ 置信區間 $=1.188-2.681 ; \mathrm{P}=0.005$ ）、肺炎（比值比 $=1.50$; $95 \%$ 置信區間 $=1.030-2.187 ; \mathrm{P}=0.034)$ ，以及碳青徽烯類耐藥 或超廣譜 $\beta$-內酰胺酶產生的分離株（比值比 $=12.51 ; 95 \%$ 置信區 間=7.886-19.487；P<0.001）與較大風險有經驗性治療不當相關。經 驗性治療不當的患者的 90 天死亡率顯著更高；獨立預測因素包括肺炎

（風險比=2.94；95\%置信區間=2.271-3.808；P $<0.001$ ）、胃腸道感染

（風險比=2.77；95\%置信區間=2.055-3.744； P<0.001）、經驗性抗 生素無效（風險比=2.45；95\%置信區間=1.928-3.124； $\mathrm{P}<0.001$ ）

年齡較大（風險比 $=1.79 ； 95 \%$ 置信區間 $=1.356-2.371 ； \mathrm{P}<0.001$ )

實體瘤（風險比 $=1.77 ； 95 \%$ 置信區間 $=1.401-2.231 ; \mathrm{P}<0.001$ ) 碳青徽烯類耐藥或超廣譜 $\beta$-內酰胺酶產生的分離株（風險比 $=1.64$; 95\%置信區間 $=1.170-2.297 ; \mathrm{P}=0.004$ ）、透過內科住院的患者 （風險比 $=1.39 ； 95 \%$ 置信區間 $=1.076-1.800 ； \mathrm{P}=0.012$ ），以及連續 器官衰竭評估總分較高（風險比=1.09；95\%置信區間=1.058-1.112； $\mathrm{P}=0.023$ ) 。在ICU患者中, 經驗性抗生素治療不當與患者使用呼吸 機時間增加和 90 天死亡率顯著相關。

結論：肺炎克雷伯菌血症與高90天死亡率和ICU死亡率相關；經驗性 抗生素治療不當可增加90天死亡率。

\section{Introduction}

Klebsiella pneumonia causes various clinically important infections. In 2017, K pneumoniae was the third most common isolate in intensive care units (ICUs) and second most common isolate in all patients in the Hong Kong East Cluster. ${ }^{1}$

The emergence of multidrug-resistant $K$ pneumoniae infections is an increasing concern. ${ }^{2}$ There have been outbreaks of extended-spectrum beta-lactamase (ESBL)-producing strains, carbapenem-resistant (CR) and carbapenemaseproducing (CP) strains, and hypervirulent $K$ pneumoniae infections both in Hong Kong and worldwide. $^{2-4}$ In Greece and Italy, CP K pneumoniae comprises $68.3 \%$ of all $K$ pneumoniae strains. ${ }^{5}$

The importance of appropriate early empirical antibiotics has been repeatedly emphasised in the management of septic shock by the Surviving Sepsis Campaign 2016. ${ }^{6}$ Previously, we highlighted the importance of appropriate early antibiotics for successful patient outcomes. ${ }^{4}$ To the best of our knowledge, risk factors for receiving inappropriate empirical antibiotics have not yet been explored. Therefore, in the present study, we aimed to evaluate the impact of appropriate empirical antibiotics on outcomes in patients with $K$ pneumoniae bacteraemia; we also performed subgroup analysis on ICU patients with K pneumoniae bacteraemia.

\section{Methods}

\section{Study design and data collection}

We conducted a retrospective analysis of adult patients with $K$ pneumoniae bacteraemia who were admitted to Pamela Youde Nethersole Eastern Hospital within the period from 1 January 2009 to 30 June 2017. Pamela Youde Nethersole Eastern Hospital is a 1700-bed hospital in Hong Kong which provides extensive services except cardiothoracic surgery, transplant surgery, and burns. Patients were excluded if they were aged $<18$ years or had incomplete information. Patient medical records were reviewed, as were data in clinical management and clinical information systems (IntelliVue Clinical Information Portfolio; Philips Medical, Amsterdam, The Netherlands). The clinical management system is a database that stores patients' demographics, laboratory results, and drug administration records for all public hospitals in Hong Kong. In the event of missing data, medical records were reviewed manually. Baseline demographics, clinical characteristics, and microbiological data for all included patients were identified from the above databases and medical records.

Disease severity was quantified using the maximum Sequential Organ Failure Assessment (SOFA) score. ${ }^{7}$ The following clinical outcome data were investigated: use of invasive organ supports (eg, inotropic use, mechanical ventilation, and renal replacement therapies), ICU and hospital lengths of stay (LOSs), ICU ventilator duration, and mortality. The primary outcome was 90-day allcause mortality; secondary outcomes were ICU and hospital mortalities, ICU and hospital LOSs, and ICU ventilator duration.

\section{Definitions}

Klebsiella pneumoniae bacteraemia was defined as the growth of $K$ pneumoniae in one or more blood cultures. If more than one positive blood culture result was recorded, only the first sample was included. Empirical antibiotic treatment was defined as the antibiotic used within 24 hours after a culture sample was collected. The empirical antibiotic treatment was considered appropriate if at least one of the antibiotic agents was consistent with the in vitro susceptibility results. ${ }^{8-10}$ Communityacquired infection was defined as $K$ pneumoniae 
identified in patients upon admission or within 48 hours after admission; hospital-acquired infection was defined as $K$ pneumoniae identified in patients at $>48$ hours after admission. ${ }^{11}$ Hepatobiliary sepsis comprised liver abscess, cholangitis, and cholecystitis; gastrointestinal sepsis comprised spontaneous bacterial peritonitis, peritonitis caused by bowel perforation, and intra-abdominal abscesses (excluding liver abscess). Medical co-morbidities (eg, diabetes mellitus, cirrhosis, congestive heart failure, chronic renal impairment, haematological malignancy and solid tumour) were defined in accordance with the International Classification of Disease coding. Prior steroid use was defined as oral or intravenous steroid consumption within 30 days before the index positive blood culture result. Chemotherapy use was defined as oral or intravenous infusion of biological agents or chemotherapy administered within 30 days before the index positive blood culture result. Any antibiotics usage within 30 days before the index hospital admission was regarded as prior antibiotics usage.

\section{Microbiology}

Blood cultures were incubated and processed. Blood culture results were considered negative if no positive growth occurred after 5 to 7 days. Susceptibility interpretation was based on Clinical and Laboratory Standards Institute interpretive criteria. The ESBL testing was based on Clinical and Laboratory Standards Institute testing criteria ${ }^{12-18}$ or the double-disk synergy test described by Jarlier et al. ${ }^{19}$ Regarding CR $K$ pneumoniae, the isolates were sent to the Public Health Laboratory Centre of Hong Kong if further genetic testing was required to confirm carbapenemase production. Multiplex real-time polymerase chain reaction assays were performed to detect Classes A, B, and D carbapenemase gene targets.

\section{Statistical analysis}

We compared the characteristics and clinical parameters between patients treated with appropriate and inappropriate empirical antibiotics, as well as between 90-day survivors and non-survivors. Results are expressed as the median \pm interquartile range (IQR) or as the number (percentage) of patients, as appropriate.

Categorical variables were compared by the Pearson Chi squared test or Fisher's exact test, as appropriate for univariate analysis. Continuous variables were compared by using the Mann-Whitney $U$ test. Variables with $\mathrm{P}<0.2$ in univariate analysis or with clinical significance from previous studies were included in the multivariate analysis. Independent predictors for 90 -day mortality were assessed by Cox regression analysis. Logistic regression analysis was used to assess independent predictors for receiving appropriate and inappropriate empirical antibiotics. Post hoc analysis was performed for patients with chronic renal failure and resistant organisms.

The Statistical Package for Social Sciences (Windows version 24.0; IBM Corp, Armonk [NY], US) was used to perform statistical analyses.

\section{Results}

\section{Baseline characteristics}

During the 8.5-year study period, we identified 984 patients with $K$ pneumoniae bacteraemia; of these, $686(69.7 \%)$ and $298(30.3 \%)$ received appropriate and inappropriate empirical antibiotics, respectively. Table 1 shows the baseline demographics of patients who received appropriate and inappropriate empirical antibiotics. The median patient age was 75 years (IQR=63-83 years). The most common types of infection were hepatobiliary tract infection (33.1\%), urosepsis (24.4\%), and respiratory tract infection (18.4\%). The overall 90-day mortality was $32.7 \%$, hospital mortality was $22.5 \%$, and median hospital LOS was 10.68 days (5.38-22.81 days, $\mathrm{P}<0.001)$.

\section{Appropriateness of empirical antibiotics}

Univariate analysis (Table 1) revealed that the risk factors for receiving inappropriate empirical antibiotics were age $>65$ years $(\mathrm{P}=0.044)$, chronic renal impairment $(\mathrm{P}<0.001)$, respiratory tract infection $(\mathrm{P}=0.002)$, mechanical ventilation $(\mathrm{P}=0.001), \mathrm{CR}$ or ESBL-producing isolates (both $\mathrm{P}<0.001)$, and higher total SOFA score $(\mathrm{P}=0.048)$. Hepatobiliary sepsis was associated with a higher rate of appropriate empirical antibiotic treatment $(\mathrm{P}=0.009)$.

Table 2 demonstrates the logistic regression analysis of the predictors for the appropriateness of empirical antibiotics for all patients and ICU subgroup. These include older patients $(\mathrm{P}=0.010)$, chronic kidney disease $(\mathrm{P}=0.007)$, mechanical ventilation $(\mathrm{P}=0.005)$, respiratory tract infection $(\mathrm{P}=0.034)$, and either carbapenem resistance or ESBL production $(\mathrm{P}<0.001)$. Table 1 shows that the 90 -day and hospital mortalities were significantly higher in patients with inappropriate empirical treatment (both $\mathrm{P}<0.001$ ). Moreover, the hospital LOS was shorter in patients who received inappropriate empirical antibiotics $(\mathrm{P}<0.001)$.

Receipt of inappropriate empirical antibiotics was associated with higher hospital mortality; this finding was consistent in ICU subgroup (56\% vs $23 \%$ ). The absolute risk increases in mortality associated with the receipt of inappropriate empirical antibiotics were $18.3 \%$ and $33 \%$ in ICU subgroup. The number of inappropriate empirical antibiotics associated with each mortality was five in the ICU subgroup and three in all patients. 
TABLE I. Clinical characteristics of adult patients with Klebsiella pneumoniae bacteraemia*

\begin{tabular}{|c|c|c|c|c|}
\hline Parameters & All patients $(n=984)$ & $\begin{array}{c}\text { Received appropriate } \\
\text { empirical antibiotics } \\
(n=686,69.7 \%)\end{array}$ & $\begin{array}{c}\text { Received inappropriate } \\
\text { or no empirical } \\
\text { antibiotics }(n=298,30.3 \%)\end{array}$ & $P$ value \\
\hline Age, y & $75(63-83)$ & $74(63-83)$ & $76(66-84)$ & 0.075 \\
\hline Age $>65$ y & $703(71.4 \%)$ & $477(69.5 \%)$ & $226(75.8 \%)$ & 0.044 \\
\hline Male sex & $581(59.0 \%)$ & 404 (58.9\%) & $177(59.4 \%)$ & 0.883 \\
\hline Admitting department & & & & 0.841 \\
\hline Medicine & $690(70.1 \%)$ & $481(70.1 \%)$ & $209(70.1 \%)$ & \\
\hline Surgery & $221(22.5 \%)$ & $151(22.0 \%)$ & $70(23.5 \%)$ & \\
\hline Oncology & $39(4.0 \%)$ & $29(4.2 \%)$ & $10(3.4 \%)$ & \\
\hline Others & $34(3.5 \%$ & $25(3.6 \%)$ & $9(3.0 \%)$ & \\
\hline \multicolumn{5}{|l|}{ Co-morbidities } \\
\hline Diabetes mellitus & $343(34.9 \%)$ & $240(35.0 \%)$ & $103(34.6 \%)$ & 0.899 \\
\hline Cirrhosis & $29(2.9 \%)$ & $17(2.5 \%)$ & $12(4.0 \%)$ & 0.187 \\
\hline $\mathrm{CHF}$ & $122(12.4 \%)$ & $77(11.2 \%)$ & $45(15.1 \%)$ & 0.090 \\
\hline CKD/ESRF & $126(12.8 \%)$ & $69(10.1 \%)$ & $57(19.1 \%)$ & $<0.001$ \\
\hline Haematological malignancy & $50(5.1 \%)$ & $32(4.7 \%)$ & $18(6.0 \%)$ & 0.367 \\
\hline Solid tumour & $339(34.5 \%)$ & $239(34.8 \%)$ & $100(33.6 \%)$ & 0.697 \\
\hline Steroid treatment (30D) & $104(10.6 \%)$ & $74(10.8 \%)$ & $30(10.1 \%)$ & 0.736 \\
\hline Chemotherapy (30D) & $39(4.0 \%)$ & 27 (3.9\%) & $12(4.0 \%)$ & 0.946 \\
\hline \multicolumn{5}{|l|}{ Source of sepsis } \\
\hline Hepatobiliary & $326(33.1 \%)$ & $245(35.7 \%)$ & $81(27.2 \%)$ & 0.009 \\
\hline Gastrointestinal & $115(11.7 \%)$ & $80(11.7 \%)$ & $35(11.7 \%)$ & 0.970 \\
\hline Respiratory & $181(18.4 \%)$ & $109(15.9 \%)$ & $72(24.2 \%)$ & 0.002 \\
\hline Urosepsis & $240(24.4 \%)$ & $164(23.9 \%)$ & $76(25.5 \%)$ & 0.592 \\
\hline Musculoskeletal & $20(2.0 \%)$ & $15(2.2 \%)$ & $5(1.7 \%)$ & 0.603 \\
\hline Neurological & $2(0.2 \%)$ & $2(0.3 \%)$ & 0 & 1.000 \\
\hline Others or unknown & $100(10.2 \%)$ & $71(10.3 \%)$ & $29(9.7 \%)$ & 0.768 \\
\hline Hospital-acquired infection & $220(22.4 \%)$ & $134(19.5 \%)$ & $86(28.9 \%)$ & $<0.001$ \\
\hline CVC usage & $194(19.7 \%)$ & $129(18.8 \%)$ & $65(21.8 \%)$ & 0.276 \\
\hline Prior antibiotic use (30D) & $772(78.5 \%)$ & $533(77.7 \%)$ & $239(80.2 \%)$ & 0.380 \\
\hline Septic shock & $256(26.0 \%)$ & $174(25.4 \%)$ & $82(27.5 \%)$ & 0.479 \\
\hline Total SOFA score & $3(2.00-6.00)$ & $3(2.00-6.00)$ & $4(2.00-7.00)$ & 0.048 \\
\hline \multicolumn{5}{|l|}{ Supportive treatment } \\
\hline RRT & $90(9.1 \%)$ & $60(8.7 \%)$ & $30(10.1 \%)$ & 0.509 \\
\hline Mechanical ventilation & $151(15.3 \%)$ & $88(12.8 \%)$ & $63(21.1 \%)$ & 0.001 \\
\hline Vasopressor & $164(16.7 \%)$ & $115(16.8 \%)$ & $49(16.4 \%)$ & 0.901 \\
\hline ICU care & 205 (20.8\%) & $148(21.6 \%)$ & $57(19.1 \%)$ & 0.385 \\
\hline \multicolumn{5}{|l|}{ Resistance } \\
\hline ESBL & $113(11.5 \%)$ & $24(3.5 \%)$ & $89(29.9 \%)$ & $<0.001$ \\
\hline Carbapenem & $20(2.0 \%)$ & $3(0.4 \%)$ & $17(5.7 \%)$ & $<0.001$ \\
\hline \multicolumn{5}{|l|}{ Outcome } \\
\hline 90-Day mortality & $322(32.7 \%)$ & $183(26.7 \%)$ & $139(46.6 \%)$ & $<0.001$ \\
\hline Hospital mortality & $221(22.5 \%)$ & $116(16.9 \%)$ & $105(35.2 \%)$ & $<0.001$ \\
\hline Hospital LOS, d & $10.68(5.38-22.81)$ & $11.25(6.02-23.5)$ & $8.94(3.25-20.12)$ & $<0.001$ \\
\hline Event-free LOS, d & $11.43(6.13-23.82)$ & $11.43(6.14-23.69)$ & $11.58(5.99-25.03)$ & 1.000 \\
\hline
\end{tabular}

Abbreviations: $30 \mathrm{D}=$ within preceding 30 days; $\mathrm{CHF}=$ congestive heart failure; $\mathrm{CKD} / \mathrm{ESRF}=$ chronic kidney disease/end-stage renal failure; CVC = central venous catheter; ESBL = extended-spectrum beta-lactamase; ICU = intensive care unit; $L O S=$ length of stay; RRT = renal replacement therapy (ie, intermittent or continuous replacement); SOFA = Sequential Organ Failure Assessment; Vasopressor = dopamine, adrenaline, and/or noradrenaline

* Data are shown as No. (\%) or median (interquartile range), unless otherwise specified 
TABLE 2. Independent predictors for receipt of inappropriate empirical antibiotics, according to logistic regression analysis

\begin{tabular}{|c|c|c|c|c|c|}
\hline \multirow[t]{2}{*}{ Variables } & \multicolumn{2}{|c|}{ All patients $(n=984)$} & \multicolumn{3}{|c|}{ ICU subgroup ( $n=205)$} \\
\hline & Odds ratio $(95 \% \mathrm{Cl})$ & $P$ value & Variables & Odds ratio $(95 \% \mathrm{Cl})$ & $P$ value \\
\hline Carbapenem or ESBL resistance & $12.51(7.886-19.487)$ & $<0.001$ & CKD/ESRF & $4.80(1.563-14.759)$ & 0.006 \\
\hline CKD/ESRF & $1.81(1.181-2.785)$ & 0.007 & ESBL or carbapenem resistance & $4.34(1.667-11.295)$ & 0.003 \\
\hline Mechanical ventilation & $1.79(1.188-2.681)$ & 0.005 & Mechanical ventilation & $4.07(1.831-9.03)$ & 0.001 \\
\hline Age $>65 y$ & $1.60(1.120-2.295)$ & 0.010 & Solid tumour & $0.38(0.154-0.944)$ & 0.037 \\
\hline Respiratory tract infection & $1.50(1.030-2.187)$ & 0.034 & & & \\
\hline
\end{tabular}

Abbreviations: 95\% Cl = 95\% confidence interval; CKD/ESRF = chronic kidney disease/end-stage renal failure; ESBL = extended-spectrum beta-lactamase; ICU = intensive care unit; SOFA = Sequential Organ Failure Assessment

Other factors included that did not reach statistical significance: cirrhosis, congestive heart failure, hepatobiliary sepsis, hospital-acquired infection, and total SOFA score $\left(\mathrm{C}\right.$ index $=0.7 \mathrm{I} 8 ; 95 \% \mathrm{Cl}=0.68 \mathrm{I}-0.755$; Hosmer-Lemeshow goodness-of-fit test, $\mathrm{c}^{2}=10.6 \mathrm{I}$; degrees of freedom $=5 ; \mathrm{P}=0.060$ )

\section{Multidrug-resistant Klebsiella pneumoniae infections}

Antibiograms showing the proportions of nonsusceptible $K$ pneumoniae isolates are described in the Online Supplementary Table 1 . Twenty $(2.0 \%)$ patients had CR $K$ pneumoniae bacteraemia, but molecular tests in the Public Health Laboratory Centre of Hong Kong revealed that none of them had CP strains. Overall, 113 (11.5\%) patients had ESBL-producing infections.

Post hoc analysis revealed that patients with chronic renal failure were more likely to have ESBL infections $(18.3 \%$ vs $10.5 \% ; \mathrm{P}=0.011)$ and $C R$ infections ( $0.056 \%$ vs $0.015 \%$; $\mathrm{P}=0.003)$.

\section{Ninety-day mortality}

The 90-day all-cause mortalities were $32.7 \%$ in all patients with $K$ pneumoniae bacteraemia and $34.6 \%$ in the ICU subgroup. Univariate analysis (Table 3) showed that 90-day non-survivors were more likely to be aged $>65$ years $(\mathrm{P}<0.001)$, admitted through the Department of Medicine $(\mathrm{P}<0.001)$, have septic shock $(\mathrm{P}=0.005)$, have a higher total SOFA score $(\mathrm{P}<0.001)$, receive inappropriate or no empirical antibiotics $(\mathrm{P}<0.001$ and $\mathrm{P}<0.001$, respectively), have solid tumour $(\mathrm{P}<0.001)$, have respiratory tract infection $(\mathrm{P}<0.001)$, be mechanically ventilated $(P<0.001)$, have gastrointestinal infections $(P<0.001)$, and require renal replacement therapy $(\mathrm{P}=0.044)$. Patients with diabetes $(\mathrm{P}=0.001)$, hepatobiliary sepsis $(\mathrm{P}<0.001)$, and urosepsis $(\mathrm{P}<0.001)$ had lower 90-day mortalities.

Table 4 shows the Cox regression analysis of predictors for 90-day mortality. Independent predictors for increased 90-day mortality were respiratory tract infection $(\mathrm{P}<0.001)$, gastrointestinal infection $(P<0.001), \quad$ inappropriate empirical antibiotics $(\mathrm{P}<0.001)$, older age $(\mathrm{P}<0.001)$, solid tumour $(\mathrm{P}<0.001)$, patients admitted through the Department of Medicine $(\mathrm{P}=0.012)$, and higher total
SOFA score $(\mathrm{P}<0.001)$. Patients with diabetes had lower 90-day mortality $(\mathrm{P}=0.001)$. The Figure shows the Kaplan-Meier survival plot and according to log rank analysis, the results demonstrated a statistically significant improvement in survival among patients who received appropriate empirical antibiotics $(\mathrm{P}<0.001)$.

\section{Intensive care unit subgroup analysis}

Online Supplementary Table 2 shows the demographics for inappropriate empirical antibiotics by logistic regression analysis in ICU subgroup. Overall, 205 (20.8\%) patients required intensive care; among them, 148 (72.2\%) received appropriate empirical antibiotics, while $57(27.8 \%)$ received inappropriate or no empirical antibiotics. The median patient age was 68 years (IQR $=58-78$ years). The commonest infection was hepatobiliary infections (42.9\%), followed by respiratory tract (23.4\%) and urosepsis (14.6\%). Furthermore, 82.4\% of the patients had septic shock, 33.2\% received renal replacement therapy, $60 \%$ received mechanical ventilation, and $78.5 \%$ had vasopressor use. The ICU and overall 90 -day mortalities were $18.5 \%$ and $34.6 \%$, respectively. The receipt of inappropriate empirical antibiotics was significantly associated with higher 90-day mortality $(59.6 \%$ vs $25.0 \%$; $\mathrm{P}<0.001)$, higher ICU mortality ( $35.1 \%$ vs $12.2 \%$; $\mathrm{P}<0.001)$, higher hospital mortality (56.1\% vs $23.0 \%$; $\mathrm{P}<0.001$ ), and longer ventilator duration ( $2 \mathrm{~d}$ vs $1 \mathrm{~d}, \mathrm{P}=0.026$ ).

Cox regression analysis showed that the receipt of inappropriate or no empirical antibiotics $(\mathrm{P}<0.001$; Table 4) was the strongest independent predictor of 90-day mortality in critically ill patients with $K$ pneumoniae bacteraemia. Other independent predictors were congestive heart failure $(\mathrm{P}=0.02)$, admitted through the Department of Medicine $(\mathrm{P}=0.016)$, and a higher total SOFA score $(\mathrm{P}<0.001)$. Ninety-day non-survivors had longer hospital LOS $(\mathrm{P}<0.001)$. 
TABLE 3. Clinical characteristics and risk factors associated with 90-day mortality

\begin{tabular}{|c|c|c|c|c|}
\hline Parameters & Total $(n=984)$ & $\begin{array}{c}\text { 90-Day non-survivors } \\
(\mathrm{n}=322,32.7 \%)\end{array}$ & $\begin{array}{c}\text { 90-Day survivors } \\
(\mathrm{n}=662,67.3 \%)\end{array}$ & $P$ value \\
\hline Age, y & $75.0(63.0-83.0)$ & $73.5(62-82)$ & $77.0(67-85)$ & 0.000 \\
\hline Age $>65 y$ & $703(71.4 \%)$ & $257(79.8 \%)$ & $446(67.4 \%)$ & $<0.001$ \\
\hline Male sex & $581(59.0 \%)$ & $185(57.5 \%)$ & $396(59.8 \%)$ & 0.479 \\
\hline Admitting department & & & & $<0.001$ \\
\hline Medicine & $690(70.1 \%)$ & $240(74.5 \%)$ & $450(68.0 \%)$ & \\
\hline Surgery & $221(22.5 \%)$ & $48(14.9 \%)$ & $173(26.1 \%)$ & \\
\hline Oncology & $39(4.0 \%)$ & $25(7.8 \%)$ & $14(2.1 \%)$ & \\
\hline Others & $34(3.5 \%)$ & $9(2.8 \%)$ & $25(3.8 \%)$ & \\
\hline \multicolumn{5}{|l|}{ Co-morbidities } \\
\hline Diabetes mellitus & $343(34.9 \%)$ & $89(27.6 \%)$ & $254(38.4 \%)$ & 0.001 \\
\hline Cirrhosis & $29(2.9 \%)$ & $12(3.7 \%)$ & $17(2.6 \%)$ & 0.313 \\
\hline $\mathrm{CHF}$ & $122(12.4 \%)$ & $43(13.4 \%)$ & $79(11.9 \%)$ & 0.526 \\
\hline CKD/ESRF & $126(12.8 \%)$ & $40(12.4 \%)$ & $86(13.0 \%)$ & 0.802 \\
\hline Haematological malignancy & $50(5.1 \%)$ & $20(6.2 \%)$ & $30(4.5 \%)$ & 0.260 \\
\hline Solid tumour & $339(34.5 \%)$ & $142(44.1 \%)$ & $197(29.8 \%)$ & $<0.001$ \\
\hline Steroid (30D) & $104(10.6 \%)$ & $35(10.9 \%)$ & $69(10.4 \%)$ & 0.831 \\
\hline Chemotherapy (30D) & $39(4.0 \%)$ & $13(4.0 \%)$ & $26(3.9 \%)$ & 0.934 \\
\hline \multicolumn{5}{|l|}{ Source of sepsis } \\
\hline Hepatobiliary & $326(33.1 \%)$ & $73(22.7 \%)$ & $253(38.2 \%)$ & $<0.001$ \\
\hline Gastrointestinal & $115(11.7 \%)$ & $61(18.9 \%)$ & $54(8.2 \%)$ & $<0.001$ \\
\hline Respiratory & $181(18.4 \%)$ & $101(31.4 \%)$ & $80(12.1 \%)$ & $<0.001$ \\
\hline Urosepsis & $240(24.4 \%)$ & $53(16.5 \%)$ & $187(28.2 \%)$ & $<0.001$ \\
\hline Musculoskeletal & $20(2.0 \%)$ & $8(2.5 \%)$ & $12(1.8 \%)$ & 0.483 \\
\hline Neurological & $2(0.2 \%)$ & $1(0.3 \%)$ & $1(0.2 \%)$ & 0.548 \\
\hline Others or unknown & $100(10.2 \%)$ & $25(7.8 \%)$ & $75(11.3 \%)$ & 0.082 \\
\hline Hospital-acquired Klebsiella pneumoniae & $220(22.4 \%)$ & $95(29.5 \%)$ & $125(18.9 \%)$ & $<0.001$ \\
\hline CVC usage & $194(19.7 \%)$ & $64(19.9 \%)$ & $130(19.6 \%)$ & 0.930 \\
\hline Prior antibiotic use (30D) & $772(78.5 \%)$ & $259(80.4 \%)$ & $513(77.5 \%)$ & 0.292 \\
\hline Septic shock & $256(26.0 \%)$ & $102(31.7 \%)$ & $154(23.3 \%)$ & 0.005 \\
\hline Total SOFA score & $3.0(2.00-6.00)$ & $4.0(2.00-8.00)$ & $3.0(2.00-5.00)$ & $<0.001$ \\
\hline \multicolumn{5}{|l|}{ Supportive treatment } \\
\hline RRT & $90(9.1 \%)$ & $38(11.8 \%)$ & $52(7.9 \%)$ & 0.044 \\
\hline Mechanical ventilation & $151(15.3 \%)$ & $70(21.7 \%)$ & $81(12.2 \%)$ & $<0.001$ \\
\hline Vasopressor & $164(16.7 \%)$ & $66(20.5 \%)$ & $98(14.8 \%)$ & 0.025 \\
\hline ICU care & $205(20.8 \%)$ & $71(22.0 \%)$ & $134(20.2 \%)$ & 0.512 \\
\hline \multicolumn{5}{|l|}{ Resistance } \\
\hline ESBL & $113(11.5 \%)$ & $43(13.4 \%)$ & $70(10.6 \%)$ & 0.199 \\
\hline Carbapenem & $20(2.0 \%)$ & $7(2.2 \%)$ & $13(2.0 \%)$ & 0.826 \\
\hline \multicolumn{5}{|l|}{ Empirical treatment } \\
\hline Appropriate & $686(69.7 \%)$ & $183(56.8 \%)$ & $503(76.0 \%)$ & $<0.001$ \\
\hline Failed & 205 (20.8\%) & $76(23.6 \%)$ & $129(19.5 \%)$ & $<0.001$ \\
\hline No antibiotic administered & $93(9.5 \%)$ & $63(19.6 \%)$ & $30(4.5 \%)$ & $<0.001$ \\
\hline \multicolumn{5}{|l|}{ Outcome } \\
\hline Hospital mortality & $221(22.5 \%)$ & $212(65.8 \%)$ & $9(1.4 \%)$ & $<0.001$ \\
\hline Hospital LOS, d & $10.68(5.38-22.81)$ & $11.43(6.13-23.82)$ & $8.80(2.92-20.44)$ & $<0.001$ \\
\hline
\end{tabular}

Abbreviations: 30D = within preceding 30 days; $\mathrm{CHF}=$ congestive heart failure; $\mathrm{CKD} / \mathrm{ESRF}=$ chronic kidney disease/end-stage renal failure; CVC = central venous catheter; ESBL = extended-spectrum beta-lactamase; ICU = intensive care unit; LOS = length of stay; RRT = renal replacement therapy (ie, intermittent or continuous replacement); SOFA = Sequential Organ Failure Assessment; Vasopressor = dopamine, adrenaline, and/or noradrenaline

* Data are shown as No. (\%) or median (interquartile range), unless otherwise specified 
TABLE 4. Independent predictors for increased 90-day mortality according to Cox regression analysis*

\begin{tabular}{|c|c|c|c|c|c|}
\hline \multirow[t]{2}{*}{ Variables } & \multicolumn{2}{|c|}{ All patients $(n=984)$} & \multicolumn{3}{|c|}{ ICU subgroup ( $n=205)$} \\
\hline & Hazard ratio $(95 \% \mathrm{Cl})$ & $P$ value & Variables & Hazard ratio $(95 \% \mathrm{Cl})$ & $P$ value \\
\hline Respiratory tract infection & $2.94(2.271-3.808)$ & $<0.001$ & $\begin{array}{l}\text { Inappropriate or no empirical } \\
\text { antibiotics }\end{array}$ & $3.00(1.856-4.854)$ & $<0.001$ \\
\hline Gastrointestinal source & $2.77(2.055-3.744)$ & $<0.001$ & Congestive heart failure & $2.28(1.141-4.561)$ & 0.020 \\
\hline $\begin{array}{l}\text { Inappropriate empirical antibiotics } \\
\text { or no antibiotics }\end{array}$ & 2.45 (1.928-3.124) & $<0.001$ & Admitting department & $1.94(1.132-3.340)$ & 0.016 \\
\hline Age $>65 y$ & 1.79 (1.356-2.371) & $<0.001$ & Total SOFA score & $1.17(1.101-1.242)$ & $<0.001$ \\
\hline Solid tumour & $1.77(1.401-2.231)$ & $<0.001$ & Diabetes mellitus & $0.39(0.221-0.696)$ & 0.001 \\
\hline $\begin{array}{l}\text { ESBL-producing or carbapenem- } \\
\text { resistant isolates }\end{array}$ & $1.64(1.170-2.297)$ & 0.004 & & & \\
\hline Admitting department & $1.39(1.076-1.800)$ & 0.012 & & & \\
\hline Total SOFA score & $1.09(1.058-1.112)$ & $<0.001$ & & & \\
\hline Diabetes mellitus & $0.65(0.505-0.830)$ & 0.001 & & & \\
\hline
\end{tabular}

Abbreviations: $95 \% \mathrm{Cl}=95 \%$ confidence interval; CKD/ESRF = chronic kidney disease/end-stage renal failure; ESBL = extended-spectrum beta-lactamase; ICU = intensive care unit; SOFA = Sequential Organ Failure Assessment

Other factors included in analysis of all patients that did not reach statistical significance: hepatobiliary sepsis, urosepsis, renal replacement therapy, mechanical ventilation, hospital-acquired infection, and septic shock. Other factors included in ICU subgroup analysis that did not reach statistical significance: age $>65$ years, CKD/ESRF, hepatobiliary sepsis, gastrointestinal sepsis, respiratory tract infection, prior antibiotic use, septic shock, renal replacement therapy, mechanical ventilation, and vasopressor use

\section{Discussion}

\section{Appropriateness of empirical antibiotics}

Among all patients in this study, 686 (69.7\%) received appropriate empirical antibiotics. Furthermore, $148(72.2 \%)$ critically ill patients received appropriate empirical antibiotics. Importantly, 93 (9.5\%) patients did not receive any empirical antibiotics. The median hospital LOS for these 93 patients was significantly shorter than the LOS for all patients in the study. We performed event-free survival analysis and found that the LOSs were similar in both groups; these findings suggested that patients who received inappropriate antibiotics had more severe disease and earlier death, leading to a shorter hospital LOS.

Micek et $\mathrm{a}^{20}$ found that prior antibiotic exposure was a risk factor for inappropriate empirical therapy. Lautenbach et $\mathrm{al}^{21}$ described a positive correlation between the total cumulative dose of antibiotics and ESBL $K$ pneumoniae infection. In our cohort, prior antibiotics exposure was not significantly correlated with inappropriate empirical antibiotic treatment; furthermore, prior antibiotics exposure was not associated with ESBL infections. We examined the presence of prior antibiotics use 30 days prior to positive blood culture results, but information regarding the total cumulative antibiotics exposure in terms of dosing and duration were not available. Other information was unavailable regarding antibiotics prescribed outside hospital settings.

Patients with chronic renal failure are reportedly more prone to developing resistant infections. ${ }^{22}$ They were at greater risk of receiving inappropriate antibiotics. Additionally, hospital-

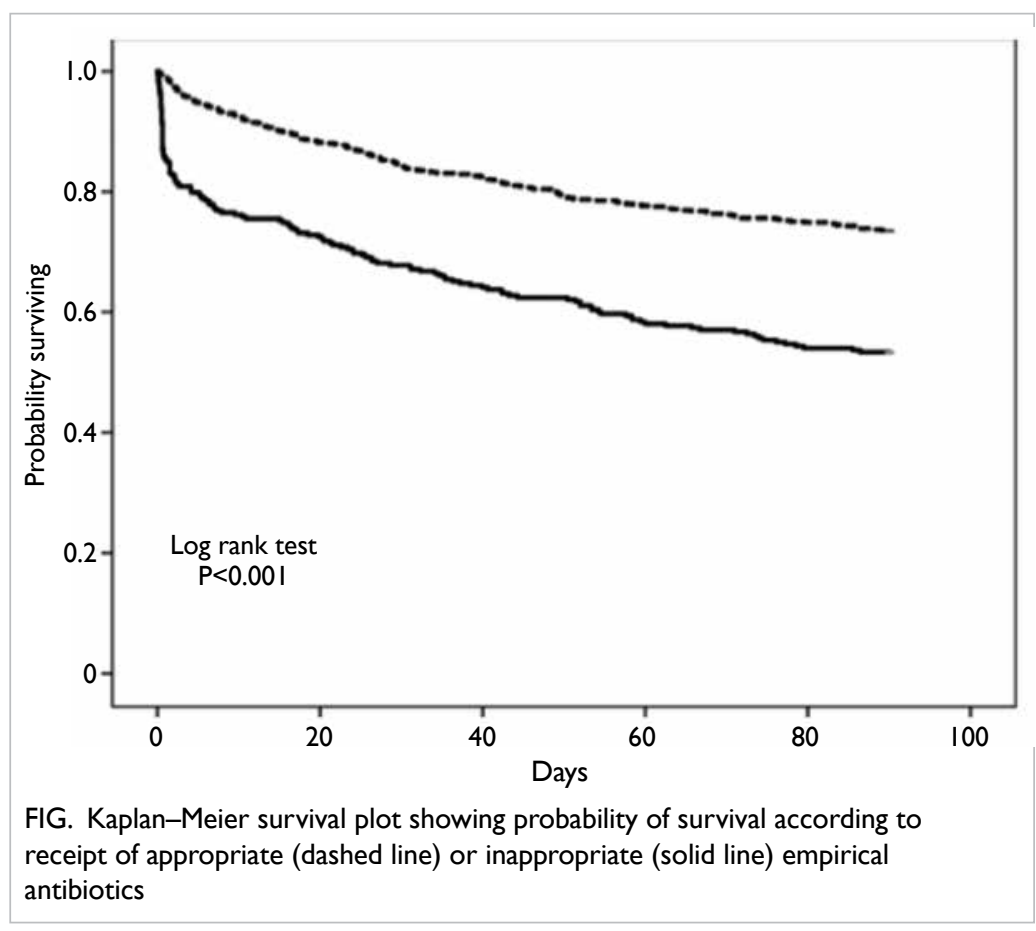

acquired infection has been associated with a higher rate of inappropriate empirical antibiotic treatment and higher 90 -day mortality. ${ }^{11}$

\section{Multidrug-resistant Klebsiella pneumoniae infections}

The rates of CR and CP K pneumoniae bacteraemia were much lower than the rates reported in 
other endemic countries. ${ }^{5}$ A study from Shanghai reported that approximately $22 \%$ of all patients with $K$ pneumoniae bacteraemia had CR infections; moreover, approximately $59 \%$ of the isolates were CR infections in patients who required intensive care. ${ }^{23}$

The prevalence of ESBL $K$ pneumoniae bacteraemia in Hong Kong is low. ${ }^{4}$ The ESBL infection rate in our cohort was $11.5 \%$, similar to previous local studies. ${ }^{1,24}$ Another study in China demonstrated a much higher rate of ESBL infections (approximately 39\%). ${ }^{25}$ Both ESBL and CR infections were not associated with increased mortality in our study in either the overall patient population or the ICU subgroup. However, ESBL and CR infections were significantly associated with longer hospital LOS.

The antibiotics sensitivity in our cohorts was comparable with the antibiogram data in the IMPACT guideline. ${ }^{1}$ Ampicillin-sulbactam or amoxicillin-clavulanate is recommended as the drug of choice for treatment of $K$ pneumomiae infections in local guidelines. ${ }^{1}$ In our cohort, more than $80 \%$ of $K$ pneumomiae isolates were susceptible to amoxicillin-clavulanate, indicating that it is a reasonable option for broad empirical coverage. Add-on therapy with aminoglycoside improves the coverage of this regimen, because more than $90 \%$ of the isolates in our cohort were sensitive to either gentamicin or amikacin. While combination therapy improves the chance of successful empirical therapy, routine use of combination therapy remains controversial. $^{6}$

\section{Ninety-day mortality}

The 90-day all-cause mortalities in our study were comparable with the findings in previous studies. ${ }^{24,26}$ Respiratory tract infections and gastrointestinal infections have consistently been associated with a worse outcome and greater mortality. ${ }^{27,28}$ In contrast, urosepsis and hepatobiliary sepsis have repeatedly associated with better survival outcomes. ${ }^{29}$ These sources of infections may be amendable to percutaneous, endoscopic, or surgical drainage, allowing more rapid and definitive control of sepsis, which leads to better survival. ${ }^{28}$

A greater proportion of patients with respiratory tract infection did not receive any empirical antibiotics, which might explain the worse outcomes in these patients. The symptoms and signs of respiratory tract infection may overlap with other diseases (eg, heart failure) and treatment may be delayed while waiting for laboratory results and imaging. Given the greater proportion of patients with respiratory tract infections who did not receive any empirical antibiotics, there is a need for early consideration of empirical antibiotics in patients with signs and symptoms of respiratory tract infections.
Consistent with the findings of previous studies, we found that older age, solid tumour, and admission through the Department of Medicine were factors associated with higher 90-day mortality. ${ }^{30,31}$ Patients with these factors were more likely to have other pre-existing co-morbidities, worse premorbid functional status, and be institutionalised. They may also have received a more conservative approach to treatment overall.

Diabetes mellitus is well-known to predispose patients to infections, such that affected patients are reportedly 4.4-fold more likely to develop bloodstream infection. ${ }^{32}$ Similar to the findings by Peralta et $\mathrm{al},{ }^{32}$ we did not find increased mortality among patients with diabetes. In our cohort, diabetic patients were more likely to had urosepsis than respiratory tract infections. Greater frequency of urosepsis and smaller frequency of respiratory tract infection may have an overall positive effect on survival. Moreover, advances in diabetes care in recent decades (eg, newer generations of medication and integrated multidisciplinary care) have led to neutral effects of diabetes on short-term mortality in patients undergoing major operations and patients with sepsis..$^{33-35}$ Glycaemic status, haemoglobin A1c levels, and diabetes severity were not available in the present study; thus, we could not delineate how diabetes control affected bacteraemia outcomes. Our results are limited to demonstrating that 90-day mortality and diabetes have a greater impact on long-term survival, although this conclusion may not be apparent in the current study. ${ }^{33}$

In addition to mortality, we demonstrated that the inappropriate use of empirical antibiotics was associated with longer ICU ventilator duration, which leads to greater costs and more extensive use of ICU resources. A large retrospective cohort from the US regarding Enterobacteriaceae infections showed that each additional day without appropriate antibiotics was associated with an increased hospital expenditure of US\$750 and an increased risk of 30-day readmission. ${ }^{36}$

To the best of our knowledge, the appropriateness of empirical antibiotics has consistently been identified as one of the strongest independent predictors of 90-day mortality in all affected patients and in critically ill patients. ${ }^{37-39}$ Kumar et $\mathrm{al}^{8}$ demonstrated a fivefold increase in mortality among patients with sepsis who received inappropriate initial antibiotics. In this study, we demonstrated a twofold increase in mortality in all patients with K pneumoniae bacteraemia, as well as a threefold increase in mortality in critically ill patients with $K$ pneumoniae bacteraemia. Furthermore, receipt of inappropriate initial antibiotics was the strongest independent predictor of 90-day mortality in the ICU subgroup. Zilberberg et $\mathrm{a}^{40}$ found that the detrimental effect of inappropriate empirical 
antibiotic treatment could not be corrected despite subsequent targeted antibiotic treatments. A metaanalysis revealed a slower rate of bacterial clearance and increased treatment failure rate when patients were administered inappropriate empirical antibiotic therapy. ${ }^{41}$ Appropriate early antibiotics allow rapid reduction of bacterial load and modulate host defences, thus alleviating some organ dysfunction. ${ }^{42}$ A more pragmatic approach would be the early administration of broader-spectrum empirical antibiotics and timely de-escalation, according to bacterial sensitivity and the patient's clinical progression.

The chain of sepsis management begins during the first encounter in the Emergency Department and in general hospital wards, rather than in the ICU. Early administration of antibiotics within 3 hours of hospital admission and aggressive sepsis care (beginning in the Emergency Department) can improve survival. ${ }^{42}$ Another prospective observational study demonstrated that the adequate use of empirical antibiotics prior to ICU admission was the strongest independent factor associated with survival. ${ }^{29}$ Extensive efforts are needed to facilitate early, appropriate use of empirical antibiotics, including the use of a current antibiogram, implementation of multidisciplinary sepsis management guidelines, and establishment of protocols among pharmacists, microbiologists, clinicians, and nurses.

\section{Strengths}

To the best of our knowledge, this is the largest study of $K$ pneumoniae bacteraemia in Hong Kong and in the Asia-Pacific region. By including both ICU and general ward patients, we achieved clarity regarding the diverse characteristics of $K$ pneumoniae bacteraemia. We also identified many potential predictors of $K$ pneumoniae bacteraemia-related mortality, based on our extensive literature review and previous publications, then tested these predictors using real-world patient data. By evaluating 90-day mortality, hospital LOS, and ventilator duration, our study more comprehensively evaluated immediate and longer-term complications of bacteraemia; it also provided information for future studies of costeffectiveness in terms of empirical antibiotics and resource utilisation. Finally, we used the maximum total SOFA score for severity assessment. This score has been repeatedly validated in determining disease severity and predicting mortality in critically ill patients. $^{43-45}$

\section{Limitations}

This retrospective study was subject to potential confounding factors, including selection bias that could not be completely eliminated from the analysis.
Notably, the results of this single-centre study may not be generalisable to other countries with higher CR or CP K pneumoniae infections. Furthermore, this study encompassed a long duration, in which the definitions of sepsis or septic shock might have changed. ${ }^{45}$ The care of patients with sepsis evolved over time, including advances in source control by percutaneous and endoscopic means that potentially improved patient survival. Nonetheless, the role of empirical antibiotics in patients with sepsis remains an essential sepsis consideration.

Antibiotic pharmacodynamics also has a fundamental role in bacteraemia treatment. In this study, we could not collect information regarding the timing of first-dose antibiotics, time to appropriate antibiotics, duration of antibiotics, or time to surgical treatments. Moreover, antibiotic stewardship and therapeutic de-escalation efforts, as well as their impacts on patient outcomes, were not assessed in the present study. Future studies may be needed concerning the prolonged infusion of beta-lactam antibiotics, use of combination therapies, duration of antibiotics, and serum monitoring of antibiotics.

\section{Conclusion}

The receipt of inappropriate empirical antibiotics led to twofold greater 90-day mortality in patients with K pneumoniae bacteraemia. In critically ill patients, inappropriate use of empirical antibiotics was the strongest independent predictor of mortality. Early identification of high-risk patients and administration of appropriate empirical antibiotics can improve patient outcomes.

\section{Author contributions}

Concept or design: MY Man, HP Shum.

Acquisition of data: MY Man, HP Shum.

Analysis or interpretation of data: MY Man, HP Shum.

Drafting of the manuscript: MY Man

Critical revision of the manuscript for important intellectual content: WW Yan.

All authors read and approved the final manuscript. All authors had full access to the data, contributed to the study, approved the final version for publication, and take responsibility for its accuracy and integrity.

\section{Conflicts of interest}

All authors have disclosed no conflicts of interest.

\section{Declaration}

This research was presented by $\mathrm{KC} \mathrm{Li}$ as an abstract at the 31st Annual Congress of the European Society of Intensive Care Medicine (ESICM), 21-24 October 2018, Paris, France.

\section{Funding/support}

This research received no specific grant from any funding agency in the public, commercial, or not-for-profit sectors. 


\section{Ethics approval}

This study was approved by the Hong Kong Easter Cluster Ethics Committee of the Hospital Authority (HKECREC2018-018). The requirement for written informed consent was waived because of the retrospective nature of the study.

\section{References}

1. Ho PL, Wu TC, Chao DV, et al, editors. Reducing bacterial resistance with IMPACT. Interhospital multi-disciplinary programme on antimicrobial chemotherapy. 5th edition. 2017. Available from: https://www.chp.gov.hk/files/pdf/ reducing_bacterial_resistance_with_impact.pdf. Accessed 7 Jun 2020.

2. Ben-David D, Kordevani R, Keller N, et al. Outcome of carbapenem resistant Klebsiella pneumoniae bloodstream infections. Clin Microbiol Infect 2012;18:54-60.

3. Wong $\mathrm{MH}$, Shum HP, Chen $\mathrm{JH}$, et al. Emergence of carbapenem-resistant hypervirulent Klebsiella pneumoniae. Lancet Infect Dis 2018;18:24.

4. Man MY, Shum HP, Chan YH, et al. Clinical predictors and outcomes of Klebsiella pneumoniae bacteraemia in a regional hospital in Hong Kong. J Hosp Infect 2017;97:3541.

5. Alicino C, Giacobbe DR, Orsi A, et al. Trends in the annual incidence of carbapenem-resistant Klebsiella pneumoniae bloodstream infections: a 8-year retrospective study in a large teaching hospital in northern Italy. BMC Infect Dis 2015;15:415.

6. Rhodes A, Evans LE, Alhazzani W, et al. Surviving sepsis campaign: international guidelines for management of sepsis and septic shock: 2016. Intensive Care Med 2017;43:304-77.

7. Ferreira FL, Bota DP, Bross A, Mélot C, Vincent JL. Serial evaluation of the SOFA score to predict outcome in critically ill patients. JAMA 2001;286:1754-8.

8. Kumar A, Ellis P, Arabi Y, et al. Initiation of inappropriate antimicrobial therapy results in a fivefold reduction of survival in human septic shock. Chest 2009;136:1237-48.

9. Vazquez-Guillamet C, Scolari M, Zilberberg MD, Shorr AF, Micek ST, Kollef M. Using the number needed to treat to assess appropriate antimicrobial therapy as a determinant of outcome in severe sepsis and septic shock. Crit Care Med 2014;42:2342-9.

10. Micek ST, Welch EC, Khan J, et al. Empiric combination antibiotic therapy is associated with improved outcome against sepsis due to Gram-negative bacteria: a retrospective analysis. Antimicrob Agents Chemother 2010;54:1742-8.

11. Juan $\mathrm{CH}$, Chuang $\mathrm{C}$, Chen $\mathrm{CH}$, Li L, Lin YT. Clinical characteristics, antimicrobial resistance and capsular types of community-acquired, healthcare-associated, and nosocomial Klebsiella pneumoniae bacteremia. Antimicrob Resist Infect Control 2019;8:1.

12. Clinical and Laboratory Standards Institute. M100-S16 Performance Standards for Antimicrobial Susceptibility Testing; Sixteenth Informational Supplement. Clinical and Laboratory Standards Institute, Wayne, PA; January 2006.

13. Clinical and Laboratory Standards Institute. M100-S17 Performance Standards for antimicrobial susceptibility testing; Seventeenth Informational Supplement. Clinical and Laboratory Standards Institute, Wayne, PA; January 2007.
14. Clinical and Laboratory Standards Institute. M100-S19 Performance Standards for antimicrobial susceptibility testing; Nineteenth Informational Supplement. Clinical and Laboratory Standards Institute, Wayne, PA; January 2009.

15. Clinical and Laboratory Standards Institute. M100-S20 Performance Standards for Antimicrobial Susceptibility Testing; Twentieth Informational Supplement. Clinical and Laboratory Standards Institute, Wayne, PA; January 2010.

16. Clinical and Laboratory Standards Institute. M100-S23 Performance Standards for Antimicrobial Susceptibility Testing; Twenty-Third Informational Supplement. Clinical and Laboratory Standards Institute, Wayne, PA; January 2013.

17. Clinical and Laboratory Standards Institute. M100-S25 Performance Standards for Antimicrobial Susceptibility Testing; Twenty-Fifth Informational Supplement. Clinical and Laboratory Standards Institute, Wayne, PA; January 2015.

18. Clinical and Laboratory Standards Institute. M100-S26 Performance Standards for Antimicrobial Susceptibility Testing; Twenty-Sixth Informational Supplement. Clinical and Laboratory Standards Institute, Wayne, PA; January 2016.

19. Jarlier V, Nicolas MH, Fournier G, Philippon A. Extended broad-spectrum beta-lactamases conferring transferable resistance to newer beta-lactam agents in Enterobacteriaceae: hospital prevalence and susceptibility patterns. Rev Infect Dis 1988;10:867-78.

20. Micek ST, Hampton N, Kollef M. Risk factors and outcomes for ineffective empiric treatment of sepsis caused by Gramnegative pathogens: stratification by onset of infection. Antimicrob Agents Chemother 2017;62:e01577-17.

21. Lautenbach E, Patel JB, Bilker WB, Edelstein PH, Fishman NO. Extended-spectrum beta-lactamase-producing Escherichia coli and Klebsiella pneumoniae: risk factors for infection and impact of resistance on outcomes. Clin Infect Dis 2001;32:1162-71.

22. Eilertson B, Cober E, Richter SS, et al. Carbapenemresistant Enterobacteriaceae infections in patients on renal replacement therapy. Open Forum Infect Dis 2017;4:ofx216.

23. Tian L, Tan R, Chen Y, et al. Epidemiology of Klebsiella pneumoniae bloodstream infections in a teaching hospital: factors related to the carbapenem resistance and patient mortality. Antimicrob Resist Infect Control 2016;5:48.

24. Pau CK, Ma FF, Ip M, You JH. Characteristics and outcomes of Klebsiella pneumoniae bacteraemia in Hong Kong. Infect Dis (Lond) 2015;47:283-8.

25. Li L, Huang H. Risk factors of mortality in bloodstream infections caused by Klebsiella pneumonia: a singlecenter retrospective study in China. Medicine (Baltimore) 2017;96:e7924.

26. Melot B, Colot J, Guerrier G. Bacteremic communityacquired infections due to Klebsiella pneumoniae: clinical and microbiological presentation in New Caledonia, 20082013. Int J Infect Dis 2015;41:29-31.

27. Russo A, Falcone M, Gutiérrez-Gutiérrez B, et al. Predictors of outcome in patients with severe sepsis or septic shock due to extended-spectrum beta-lactamase-producing Enterobacteriaceae. Int J Antimicrob Agents 2018;52:57785.

28. Zilberberg MD, Shorr AF, Micek ST, Vazquez-Guillamet C, Kollef MH. Multi-drug resistance, inappropriate initial 
antibiotic therapy and mortality in Gram-negative severe sepsis and septic shock: a retrospective cohort study. Crit Care 2014;18:596.

29. Garnacho-Montero J, Gutiérrez-Pizarraya A, EscorescaOrtega A, Fernández-Delgado E, López-Sánchez JM. Adequate antibiotic therapy prior to ICU admission in patients with severe sepsis and septic shock reduces hospital mortality. Crit Care 2015;19:302.

30. Antonio M, Gudiol C, Royo-Cebrecos C, Grillo S, Ardanuy $\mathrm{C}$, Carratalà J. Current etiology, clinical features and outcomes of bacteremia in older patients with solid tumors. J Geriatr Oncol 2019;10:246-51.

31. Tseng CP, Wu HS, Wu TH, Lin YT, Fung CP. Clinical characteristics and outcome of patients with communityonset Klebsiella pneumoniae bacteremia requiring intensive care. J Microbiol Immunol Infect 2013;46:217-23.

32. Peralta G, Sánchez MB, Roiz MP, Garrido JC, Teira R, Mateos F. Diabetes does not affect outcome in patients with Enterobacteriaceae bacteremia. BMC Infect Dis 2009;9:94.

33. Filsoufi F, Rahmanian PB, Castillo JG, Mechanick JI, Sharma SK, Adams DH. Diabetes is not a risk factor for hospital mortality following contemporary coronary artery bypass grafting. Interact Cardiovasc Thorac Surg 2007;6:753-8.

34. Teo BJ, Chong HC, Yeo W, Tan AH. The impact of diabetes on patient outcomes after total knee arthroplasty in an Asian population. J Arthroplasty 2018;33:3186-9.

35. Stegenga ME, Vincent JL, Vail GM, et al. Diabetes does not alter mortality or hemostatic and inflammatory responses in patients with severe sepsis. Crit Care Med 2010;38:53945.

36. Zilberberg MD, Nathanson BH, Sulham K, Fan W, Shorr AF. 30-day readmission, antibiotics costs and costs of delay to adequate treatment of Enterobacteriaceae UTI, pneumonia, and sepsis: a retrospective cohort study. Antimicrob Resist Infect Control 2017;6:124.

37. Gutiérrez-Gutiérrez B, Salamanca E, de Cueto M, et al.
Effect of appropriate combination therapy on mortality of patients with bloodstream infections due to carbapenemase-producing Enterobacteriaceae (INCREMENT): a retrospective cohort study. Lancet Infect Dis 2017;17:726-34.

38. Cheng WL, Hsueh PR, Lee CC, et al. Bacteremic pneumonia caused by extended-spectrum beta-lactamaseproducing Escherichia coli and Klebsiella pneumoniae: appropriateness of empirical treatment matters. J Microbiol Immunol Infect 2016;49:208-15.

39. Kohler PP, Volling C, Green K, Uleryk EM, Shah PS, McGeer A. Carbapenem resistance, initial antibiotic therapy, and mortality in Klebsiella pneumoniae bacteremia: a systematic review and meta-analysis. Infect Control Hosp Epidemiol 2017;38:1319-28.

40. Zilberberg MD, Shorr AF, Micek ST, Mody SH, Kollef MH. Antimicrobial therapy escalation and hospital mortality among patients with health-care-associated pneumonia: a single-center experience. Chest 2008;134:963-8.

41. Raman G, Avendano E, Berger S, Menon V. Appropriate initial antibiotic therapy in hospitalized patients with gram-negative infections: systematic review and metaanalysis. BMC Infect Dis 2015;15:395.

42. Seymour CW, Gesten F, Prescott HC, et al. Time to treatment and mortality during mandated emergency care for sepsis. N Engl J Med 2017;376:2235-44.

43. Moreno R, Vincent JL, Matos R, et al. The use of maximum SOFA score to quantify organ dysfunction/failure in intensive care. Results of a prospective, multicentre study. Working Group on Sepsis related Problems of the ESICM. Intensive Care Med 1999;25:686-96.

44. Minne L, Abu-Hanna A, de Jonge E. Evaluation of SOFAbased models for predicting mortality in the ICU: a systematic review. Crit Care 2008;12:R161.

45. Singer M, Deutschman CS, Seymour CW, et al. The third international consensus definitions for sepsis and septic shock (sepsis-3). JAMA 2016;315:801-10. 